\title{
LAG-3 expression in the inflammatory microenvironment of glioma
}

\author{
Maximilian J. Mair ${ }^{1} \cdot$ Barbara Kiesel $^{2} \cdot$ Katharina Feldmann $^{1} \cdot$ Georg Widhalm $^{2} \cdot$ Karin Dieckmann $^{3}$. \\ Adelheid Wöhrer $^{4} \cdot$ Leonhard Müllauer $^{5} \cdot$ Matthias Preusser $^{1} \cdot$ Anna S. Berghoff ${ }^{1}$ (i)
}

Received: 27 January 2021 / Accepted: 15 February 2021 / Published online: 2 March 2021

(c) The Author(s) 2021

\begin{abstract}
Purpose Immune modulatory therapies including immune checkpoint inhibitors have so far failed to result in clinically meaningful efficacy in glioma. We aimed to investigate lymphocyte activation gene 3 (LAG-3), an inhibitory receptor on immune cells and target of second-generation immune checkpoint inhibitors, in glioma.

Methods 97 patients with diffuse glioma (68 with glioblastoma, 29 with WHO grade II-III glioma) were identified from the Neuro-Biobank of the Medical University of Vienna. LAG-3 expression in the inflammatory microenvironment was assessed by immunohistochemistry (monoclonal anti-LAG-3 antibody, clone 17B4) and correlated to CD3+, CD8+, CD20+and PD-1+tumor-infiltrating lymphocytes (TILs) and PD-L1 expression on tumor cells.

Results LAG-3+ TILs could be observed in 10/97 (10.3\%) IDH-wildtype samples and in none of the included IDH-mutant glioma samples $(\mathrm{p}=0.057)$. Further, LAG-3+TILs were only observed in WHO grade IV glioblastoma, while none of the investigated WHO grade II-III glioma presented with LAG-3+ TILs $(\mathrm{p}=0.03)$. No association of O6-methylguanine-DNAmethyltransferase (MGMT) promoter methylation and presence of LAG-3+TILs was observed $(p=0.726)$. LAG-3 expression was associated with the presence of CD3+ $(\mathrm{p}=0.029), \mathrm{CD} 8+(\mathrm{p}=0.001), \mathrm{PD}-1+(\mathrm{p}<0.001)$ TILs and PD-L1+ tumor cells $(\mathrm{p}=0.021)$, respectively. No association of overall survival with LAG-3+ TIL infiltration was evident (median OS 9.9 vs. 14.2 months, $\mathrm{p}=0.95)$.

Conclusions LAG-3 is only rarely expressed on TILs in IDH-wildtype glioma and associated with active inflammatory milieu as defined by higher TIL density. Immune microenvironment diversity should be considered in the design of future immunotherapy trials in glioma.
\end{abstract}

Keywords Glioma $\cdot$ Glioblastoma $\cdot$ Tumor microenvironment $\cdot$ LAG-3 $\cdot$ Immune checkpoint

Anna S. Berghoff

anna.berghoff@meduniwien.ac.at

1 Division of Oncology and Christian Doppler Laboratory for Personalized Immunotherapy, Department of Medicine I, Medical University of Vienna, Waehringer Guertel 18-20, 1090 Vienna, Austria

2 Department of Neurosurgery, Medical University of Vienna, Vienna, Austria

3 Department of Radiation Oncology, Medical University of Vienna, Vienna, Austria

4 Division of Neuropathology and Neurochemistry, Department of Neurology, Medical University of Vienna, Vienna, Austria

5 Department of Pathology, Medical University of Vienna, Vienna, Austria

\section{Introduction}

Immune checkpoint inhibitors are a major breakthrough in oncology as durable responses can be observed in a variety of solid malignancies. However, despite the efficacy in secondary brain tumors [1-3], no clinical benefit was so far observed in primary brain tumors such as glioma. The CheckMate-143 trial comparing the anti-PD-L1 agent nivolumab to bevacizumab in recurrent glioblastoma did not meet its primary endpoint, as overall survival was comparable in both treatment arms [4]. Similarly, two trials investigating the activity of PD-1 inhibitors in newly diagnosed glioblastoma failed to meet their primary endpoints according to recently published press releases, although the final publications are pending $[5,6]$. Interestingly, membranous PD-L1 expression was observed in 37.6-61\% of human glioblastoma samples, while diffuse/fibrillary PD-L1 expression 
was seen in $88.0 \%$ of patients, indicating that the target is present in the majority of patients [7,8]. Still, the objective response rate of $7.8 \%$ towards nivolumab treatment was limited in recurrent glioblastoma [4], underlining the need to explore new immune modulatory treatment targets.

Lymphocyte activation gene 3 (LAG-3) is an inhibitory receptor which is mainly found on activated immune cells [9]. Frequently co-expressed with PD-1 on exhausted T cells, LAG-3 has become an interesting target for immune-modulating agents either alone or in combination with inhibitors of the PD-1/PD-L1 axis [10]. Anti-LAG-3 agents are under investigation in phase I-III trials in a wide array of solid tumors including lung, gastric, head and neck, hepatocellular and renal cancer as well as lymphoma and melanoma [11]. In the latter, LAG-3 expression was associated with a higher objective response rate towards LAG-3 blockade in early data of a phase I/IIA trial [12]; however, the predictive value of LAG-3 expression towards response to LAG-3 immune checkpoint inhibitors is still not fully elucidated.

In glioma, a preclinical study using a syngenic mouse model showed that treatment with anti-LAG-3 antibodies either alone or in combination with PD-1 inhibition is effective and results in prolonged survival [13]. However, systematic data on LAG-3 expression in human glioma tissue are missing so far. Therefore, the aim of this study was to evaluate LAG-3 expression in the tumor microenvironment of adult glioblastoma and WHO grade II-III glioma cases.

\section{Materials and methods}

\section{Patient cohort}

Patients aged $\geq 18$ years at diagnosis who were treated for WHO grade II-III glioma or glioblastoma were identified from the Neuro-Biobank of the Medical University of Vienna. Histological diagnosis was performed by a boardcertified neuropathologist according to the 2016 WHO Classification of Central Nervous Tumours [14]. Patient data were stored in a password-secured database (FileMaker Pro Advanced/Server 17, FileMaker Inc., Santa Clara, USA) and were handled anonymously. This study was performed according to the ethical standards of the Ethics Committee of the Medical University of Vienna (approval no. 1166/2019) and the Helsinki Declaration of 1964 with all its amendments.

\section{Immunohistochemistry}

Immunohistochemical analysis was executed using a Ventana Benchmark Ultra immunostainer (Roche Ventana Medical Systems, Tucson, AZ, USA) as described previously [15]. Used antibodies are listed in Supplementary Table 1.
Analysis of CD3+, CD8+, CD20+ and PD-1+ TILs as well as PD-L1 expression on tumor cells was evaluated semiquantitatively and available from previous publications $[8$, 16]. LAG-3+TIL were also evaluated semiquantitatively by overall impression at low microscopic magnification $(\times 100)$. Further, accumulation of TILs in predefined areas (within the viable tumor tissue, in the perivascular region and, if applicable, in the invasion zone to the surrounding brain parenchyma) was analyzed as higher magnification $(\times 200-\times 400)$. Previously published criteria were used to describe TIL density as sparse, moderate, dense or very dense $[8,16]$.

\section{Statistical analysis}

Independence of categorical variables was assessed using Chi-square or Fisher's exact test as appropriate. Overall survival (OS) was defined as the time between first surgery and all-cause death and was compared applying the log-rank test. Results were considered significant at a $p$ value of $\leq 0.05$. Due to the hypothesis-generating study design, no correction for multiple testing was applied [17].

Statistical analysis was performed using GraphPad Prism 8 (La Jolla, CA, USA), R 3.6.1 (The R Foundation for Statistical Computing, Vienna, Austria) with RStudio 1.2.1335 (RStudio Inc., Boston, MA, USA) and the packages "ggplot2" (version 3.2.0), "GGally" (version 1.4.0), "VennDiagram" (version 1.6.20), "survival" (version 2.44-1.1) and "ggpubr" (version 0.2.3).

\section{Results}

\section{Patients' characteristics}

97 patients were included, including 68/97 (70.1\%) patients with glioblastoma and 29/97 (29.9\%) with WHO grade II-III glioma. Isocitrate dehydrogenase (IDH) mutations could be detected in 27/97 (27.8\%) of included samples, while 70/97 (72.2\%) specimens were IDH wildtype (IDH-wt). Further baseline characteristics of the studied cohort are given in Table 1.

\section{LAG-3 expression on tumor-infiltrating lymphocytes}

LAG-3 expression on TILs in glioma was observed in 10/97 $(10.3 \%)$ cases (Fig. 1; tonsil as positive control shown in Fig. 1a). As previously described with other TIL subsets [16], also LAG-3+ TILs accumulated in the perivascular region, whereas the density of LAG-3+ TILs was sparse in tumor tissue (Fig. 1b/c).

LAG-3+ TILs were observed in 10/68 (14.7\%) of glioblastoma samples while no tissue specimen of WHO grade 
Table 1 Patients' characteristics

\begin{tabular}{|c|c|}
\hline & $\mathrm{n}=97$ \\
\hline \multicolumn{2}{|l|}{ Gender } \\
\hline Male & $56(57.7 \%)$ \\
\hline Female & $41(42.3 \%)$ \\
\hline Median age at diagnosis (range) & $55(25-80)$ \\
\hline \multicolumn{2}{|l|}{ WHO grade } \\
\hline WHO II & $21(21.7 \%)$ \\
\hline WHO III & $8(8.2 \%)$ \\
\hline WHO IV & $68(70.1 \%)$ \\
\hline \multicolumn{2}{|l|}{ IDH mutation } \\
\hline IDH mutated & $27(27.8 \%)$ \\
\hline IDH wildtype & $70(72.2 \%)$ \\
\hline \multicolumn{2}{|l|}{ MGMT promoter methylation } \\
\hline Methylated & $23(23.7 \%)$ \\
\hline Unmethylated & $36(37.1 \%)$ \\
\hline Unknown & $38(39.2 \%)$ \\
\hline \multicolumn{2}{|l|}{ Diagnosis according to WHO 2016 classification } \\
\hline Diffuse astrocytoma, IDH-mt & $11(11.3 \%)$ \\
\hline Gemistocytic astrocytoma, IDH-mt & $1(1.0 \%)$ \\
\hline Anaplastic astrocytoma, IDH-mt & $2(2.1 \%)$ \\
\hline Diffuse astrocytoma, IDH-wt & $2(2.1 \%)$ \\
\hline Oligodendroglioma, IDH-mt, 1p19q-codeleted & $7(7.2 \%)$ \\
\hline $\begin{array}{l}\text { Anaplastic oligodendroglioma, IDH-mt, 1p19q-code- } \\
\text { leted }\end{array}$ & $6(6.2 \%)$ \\
\hline Glioblastoma, IDH-wt & $68(69.4 \%)$ \\
\hline
\end{tabular}

II-III gliomas showed LAG-3+ TIL infiltration $(\mathrm{p}=0.03$, Fisher's exact test). Similarly, all LAG-3+ samples were from IDH-wildtype tumors $(10 / 70,14.3 \%)$ while no LAG$3+$ TILs could be found in IDH-mutant glioma $(p=0.057$, Fisher's exact test). In terms of O6-methylguanine-DNAmethyltransferase (MGMT) promoter methylation, 3/23 (13.0\%) MGMT promoter-methylated tumors showed LAG$3+$ TIL infiltration as compared to 7/36 (19.4\%) unmethylated specimen ( $p=0.726$, Fisher's exact test; Fig. 2).

There were no associations of LAG-3+ TIL infiltration with age at first surgery $(\mathrm{p}=0.135$, Mann-Whitney-U test $)$ and gender $(p=0.183$, Fisher's exact test $)$.

\section{Correlation of LAG-3+ TILs with other TIL subsets}

CD3+ TILs were observed in 65/97 (67.0\%) samples, while CD8 + TILs and PD-1 + TILs were seen in 39/97 (40.2\%) and 14/97 (14.4\%) specimen, respectively. Furthermore, PD-L1 expression on tumor cells was detected in $43 / 97$ $(44.3 \%)$ tumors.

Only samples with CD3+ TIL infiltration were also positive for LAG-3+ TILs (10/65 CD3+ vs. 0/32 CD3-; $\mathrm{p}=0.029$, Fisher's exact test; Table 2A). In 9/30 (30.0\%) CD8+ samples also LAG-3+ TIL infiltration was observed, while only $1 / 58$ (1.7\%) of CD8- specimen showed LAG$3+$ TIL infiltration ( $\mathrm{p}=0.001$, Fisher's exact test). $7 / 14$ (50.0\%) PD-1+ specimen showed LAG-3+ TIL infiltration while only $3 / 83(3.6 \%)$ PD- $1-$ samples presented with LAG-3+ TILs ( $p<0.001$, Fisher's exact test). An association between PD-L1 expression on tumor cells and LAG-3+ TIL infiltration was observed, as 8/43 (18.6\%) samples with membranous PD-L1 expression on tumor cells were infiltrated by LAG-3+ lymphocytes in contrast to only $2 / 54(3.7 \%)$ in PD-L1 - cases $(p=0.021$, Fisher's exact test). A Venn diagram in Fig. 3 illustrates the cooccurrence of CD3+, CD8+, PD-1+ and LAG-3+ TILs as well as PD-L1+tumor cells in the overall cohort. Of note, 21/97 (21.6\%) cases did show neither of the analyzed immune cell subsets.

\section{Correlation of LAG-3+ TILs with CD3+, CD8+, CD20+, PD-1+TIL infiltration and PD-L1 expression on tumor cells in the IDH-wt glioblastoma cohort}

In IDH-wt glioblastoma, CD3+ TILs were observed in $48 / 68(70.6 \%)$ samples, while CD8+ TILs were seen in $28 / 68$ (41.2\%), CD20+in 18/68 (26.5\%) and PD-1+ TILs in $14 / 68(20.6 \%)$ samples. PD-L1 expression on tumor cells was seen in 40/68 (58.8\%) cases.

Again, only samples with CD3+ TIL infiltration had LAG-3+ TILs (10/48 CD3+ vs. 0/20 CD3-; $p=0.028$, Fisher's exact test, Table 2B). In 9/28 (32.1\%) CD8+ cases LAG-3+ TIL infiltration was seen, while only $1 / 40(2.5 \%)$ of CD8- samples had LAG-3+ TILs ( $p<0.001$, Fisher's exact test). Similarly, in 6/18 (33.3\%) CD20+tumors an infiltration with LAG-3+TILs was observed, while this was only the case in $4 / 46(8.7 \%)$ CD20- samples $(p=0.017$, Fisher's exact test). Moreover, 7/14 (50.0\%) of tumors with PD-1+immune cell infiltration showed LAG-3+ TILs, while in only 3/54 (5.6\%) PD-1- tumors an infiltration with LAG-3+ lymphocytes was seen ( $p<0.001$, Fisher's exact test). However, no correlation between membranous PD-L1 expression on tumor cells and LAG$3+$ TILs could be detected (8/40 (20.0\%) PD-1+vs. $2 / 28$ $(7.1 \%) ; p=0.179$, Fisher's exact test).

\section{Prognostic impact of LAG-3+ TILs in glioblastoma}

As only IDH-wt glioblastoma cases had LAG-3+TILs, survival analysis according to LAG-3 expression was only performed in this subgroup to exclude the prognostic impact of different glioma subgroups. However, there was no significant difference in overall survival between patients with and without LAG-3+ TIL infiltration (median OS 9.9 months vs. 14.2 months; $p=0.95$, log-rank test; Fig. 4). 
Fig. 1 LAG-3 staining in a tonsil as positive control, b perivascular region and $\mathbf{c}$ tumor tissue in glioma. Magnification $\times 200$, scale bar $100 \mu \mathrm{m}$

\section{Discussion}

Here, we investigated LAG-3 expression in the inflammatory microenvironment of glioma. We found that only a small subset of tissue samples exhibited sparse infiltration by LAG-3+TILs, and all of them were IDH-wt glioblastoma cases. Overlap of LAG-3+ TILs infiltration with other immune markers was observed, underscoring that LAG-3 is expressed in samples with a particularly active immune microenvironment. The present study therefore underlines the diversity of the immune microenvironment composition in glioma. Future studies on immune modulating therapies should address the immune microenvironment diversity of glioma.

In our cohort, only $\sim 10 \%$ of samples showed sparse LAG$3+$ TIL infiltration. In line with our results, a transcriptomic study based on The Cancer Genome Atlas (TCGA) data showed that glioblastomas (and even more distinctly lowgrade gliomas) were among the tumors with the lowest expression of LAG-3 as compared to other malignancies [18]. In contrast, Harris-Bookman et al. observed that $66 \%$ of glioblastoma samples in a small cohort of 9 cases displayed variable LAG-3 expression on perivascular lymphocytes while no WHO grade II-III glioma or IDH-mt cases were analyzed [13].

LAG-3+ TIL infiltration was associated with CD3+, CD8+ and PD-1+ TILs as well as PD-L1+ tumor cells and therefore more frequently observed in specimens with an active inflammatory microenvironment. Indeed, previous studies in ovarian cancer also suggested a correlation of LAG-3+ TIL density with PD-1+ TIL density and therefore a marker for increased cancer-immune system interaction [19]. Our data therefore underline the heterogenous composition of the glioma inflammatory microenvironment cohort also in terms of LAG-3+ TIL density. The so far conducted clinical trials on LAG-3 immune checkpoint inhibitor inhibition did not require a particular biomarker. However, previous studies from extracranial tumors have repetitively underscored that an active inflammatory microenvironment is needed for a clinically meaningful tumor response [20]. Indeed, the combination of anti-PD-1 with anti-LAG-3 antibodies has not been not shown to be superior than either treatment alone in a syngeneic glioma mouse model [13]. However, no further analysis on the inflammatory microenvironment or the presence of LAG-3+TILs was included. A phase I trial is currently investigating the combination of nivolumab with the anti-LAG-3 agent relatlimab (NCT02658981) in recurrent glioblastoma. In the interim analysis, the median OS in the LAG-3 only and combination
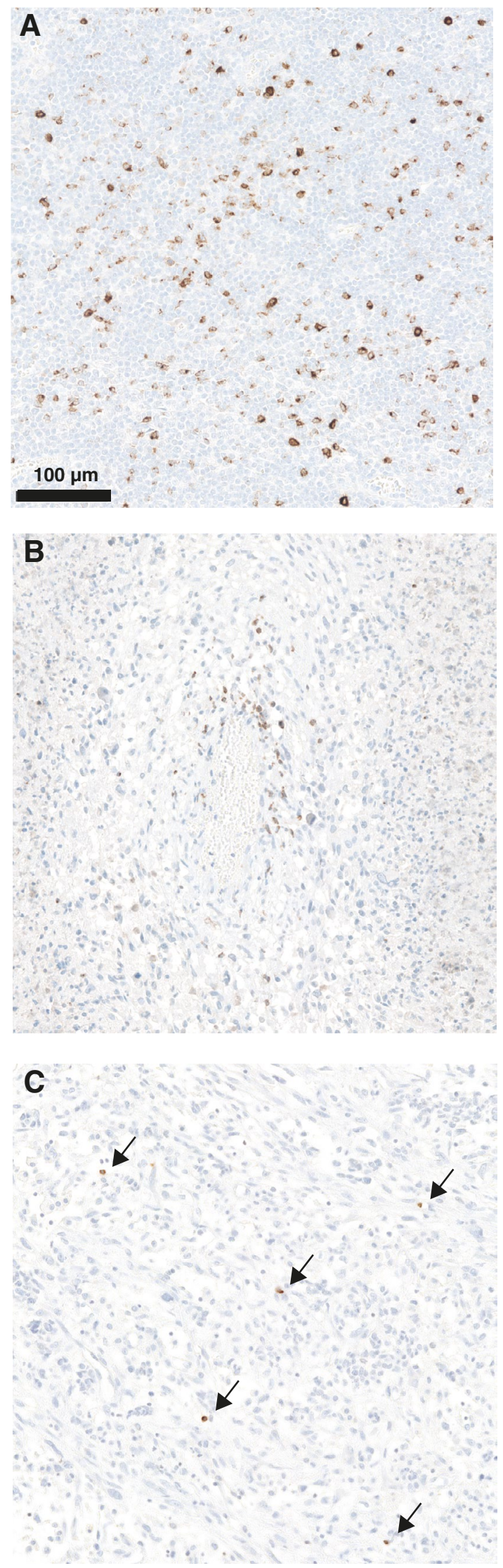
A

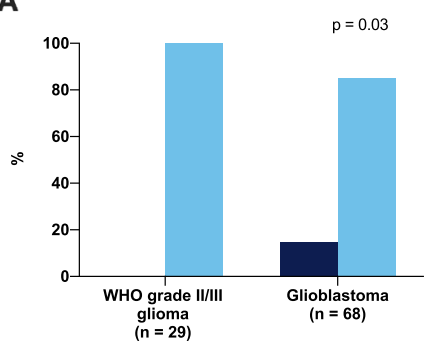

B

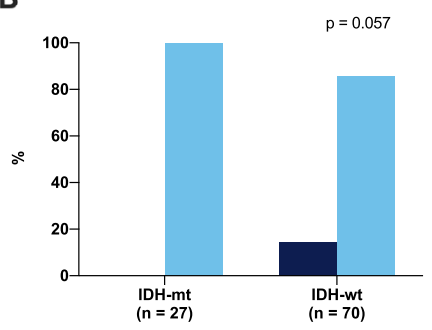

C

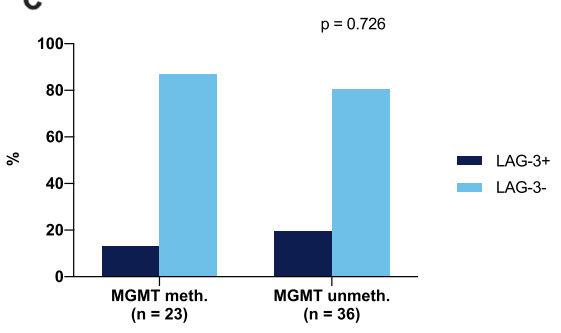

Fig. 2 Fractions of LAG-3+ cases according to a WHO grade, b IDH mutational status and $\mathbf{c}$ MGMT promoter methylation status. P-values as determined by Fisher's exact test

Table 2 Association between LAG-3 expression and other tumor-infiltrating lymphocyte subsets in (A) the overall cohort and (B) the IDH-wt glioblastoma cohort

\begin{tabular}{|c|c|c|c|c|}
\hline \multirow[t]{2}{*}{ (A) } & \multirow{2}{*}{$\begin{array}{l}\text { Overall cohort } \\
n=97\end{array}$} & \multicolumn{2}{|l|}{ LAG-3 } & \\
\hline & & + & - & \\
\hline \multirow[t]{2}{*}{ CD3 } & + & 10 & 55 & $p=0.029$ \\
\hline & - & 0 & 32 & \\
\hline \multirow[t]{2}{*}{ CD8 } & + & 9 & 30 & $\mathrm{p}=\mathbf{0 . 0 0 1}$ \\
\hline & - & 1 & 57 & \\
\hline \multirow[t]{2}{*}{ PD-1 } & + & 7 & 7 & $\mathrm{p}<0.001$ \\
\hline & - & 3 & 80 & \\
\hline \multirow[t]{3}{*}{ PD-L1 } & + & 8 & 35 & $p=0.021$ \\
\hline & - & 2 & 52 & \\
\hline & Median (range) & $1 \%(0-70 \%)$ & $0 \%(0-60 \%)$ & $\mathrm{p}=0.052$ \\
\hline \multirow[t]{2}{*}{ (B) } & IDH-wt glioblastoma & \multicolumn{2}{|l|}{ LAG-3 } & \\
\hline & $\mathrm{n}=68$ & + & - & \\
\hline \multirow[t]{2}{*}{ CD3 } & + & 10 & 38 & $p=0.020$ \\
\hline & - & 0 & 20 & \\
\hline \multirow[t]{2}{*}{ CD8 } & + & 9 & 19 & $\mathrm{p}<\mathbf{0 . 0 0 1}$ \\
\hline & - & 1 & 39 & \\
\hline \multirow[t]{2}{*}{$\mathrm{CD} 20$} & + & 6 & 12 & $p=0.017$ \\
\hline & - & 4 & 46 & \\
\hline \multirow[t]{2}{*}{ PD-1 } & + & 7 & 7 & $\mathbf{p}<\mathbf{0 . 0 0 1}$ \\
\hline & - & 3 & 51 & \\
\hline \multirow[t]{3}{*}{ PD-L1 } & + & 8 & 32 & $\mathrm{p}=0.179$ \\
\hline & - & 2 & 26 & \\
\hline & Median (range) & $1 \%(0-70 \%)$ & $1 \%(0-60 \%)$ & $p=0.436$ \\
\hline
\end{tabular}

P-values as determined by Fisher's exact test

Bold $\mathrm{p}$-values indicate $\mathrm{p} \leq 0.05$

arms was 8.5 and 8 months, respectively [21]. However, with 7 still living patients in the combination arm and 3 out of 7 with a survival beyond 20 months, durable responses might be seen in a small subset of patients. Nevertheless, also in this preliminary trial no inflammatory biomarkers were mandatory for inclusion and resistance of immunologically cold tumors could bias the result. Indeed, in extracranial tumors, LAG-3 expression in melanoma was shown to be associated with better prognosis and response to immune checkpoint inhibitors [22]; however, further translational data from trials investigating LAG-3-blocking agents in solid tumors are needed.

Our study has several limitations. First, the retrospective design is inherently linked to heterogenous baseline characteristics in the studied cohort. Furthermore, only few patients with WHO grade II-III glioma and 


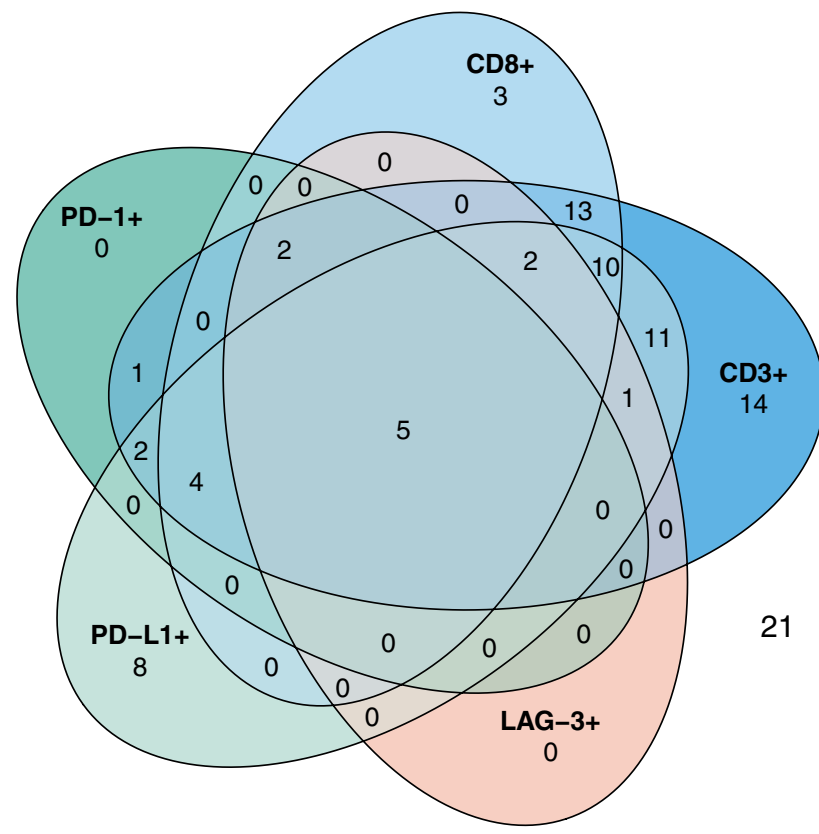

Fig. 3 Venn diagram showing the concordance of tissue-based immune markers in the included samples of the overall cohort

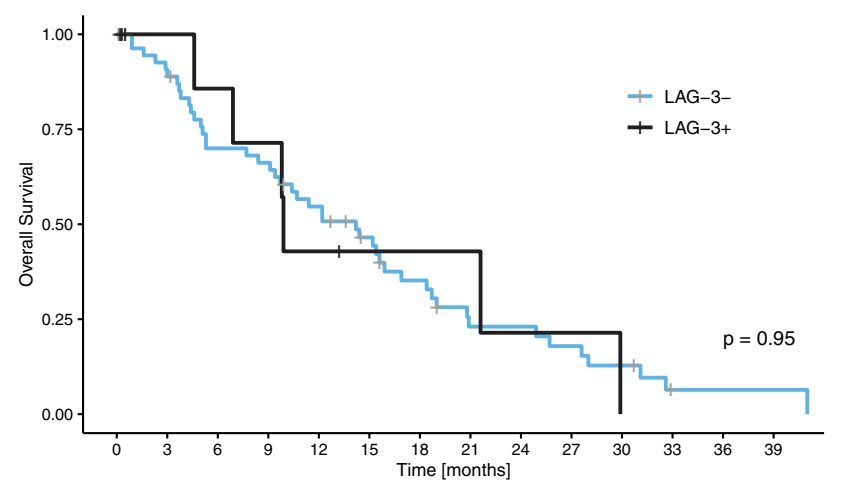

Fig. 4 Overall survival in the glioblastoma cohort according to LAG-3 expression in the tumor microenvironment

IDH-mutated glioma could be included which impeded further testing for statistical effects in these subgroups. Due to the low rate of LAG-3+ samples, further correlative analyses could not be performed due to limited statistical power. Still, data on LAG-3 expression in glioma patients is rare and the here presented cohort is among the largest published so far.

In conclusion, LAG-3 is only expressed in a small number of human glioma samples. The expression of LAG-3+in the inflammatory microenvironment shows considerable heterogeneity that needs to be acknowledged in clinical trial planning. Further profiling of tumor - immune system interactions is warranted in glioma, as only a small subset of patients responded in so far conducted immunotherapy trials and reliable biomarkers to identify benefitting patients from immune modulatory treatment modalities including LAG-3 blockade are still needed.

Supplementary Information The online version contains supplementary material available at https://doi.org/10.1007/s11060-021-03721-x.

Acknowledgements We thank Irene Erber and Stefan Traint for excellent technical assistance. This study was conducted as a part of the $\mathrm{PhD}$ thesis project "Clinical and immunological characteristics associated with lower-grade glioma prognosis" of Maximilian Mair within the Clinical Neurosciences (CLINS) program at the Medical University of Vienna. Parts of this manuscript were presented as a poster presentation at the American Society of Clinical Oncology (ASCO) Virtual Meeting 2020.

Author contributions Contribution to study design and its implementation: MJM, BK, KF, GW, LM, MP, ASB; Data analysis and interpretation: MJM, BK, KF, GW, KD, AW, LM, MP, ASB; Manuscript writing and editing: MJM, BK, KF, GW, KD, AW, LM, MP, ASB. All authors read and approved the final version of the manuscript.

Funding Open access funding provided by Medical University of Vienna. The financial support by the Austrian Federal Ministry for Digital and Economic Affairs, the National Foundation for Research, Technology and Development and the Christian Doppler Research Association is gratefully acknowledged.

\section{Declarations}

Conflict of interest Matthias Preusser has received honoraria for lectures, consultation or advisory board participation from the following for-profit companies: Bayer, Bristol-Myers Squibb, Novartis, Gerson Lehrman Group (GLG), CMC Contrast, GlaxoSmithKline, Mundipharma, Roche, BMJ Journals, MedMedia, Astra Zeneca, AbbVie, Lilly, Medahead, Daiichi Sankyo, Sanofi, Merck Sharp \& Dome, Tocagen. The following for-profit companies have supported clinical trials and contracted research conducted by MP with payments made to his institution: Boehringer-Ingelheim, Bristol-Myers Squibb, Roche, Daiichi Sankyo, Merck Sharp \& Dome, Novocure, GlaxoSmithKline, AbbVie. Anna Sophie Berghoff has received research support from Daiichi Sankyo, Roche, and honoraria for lectures, consultation or advisory board participation from Roche, Bristol-Meyers Squibb, Merck, Daiichi Sankyo as well as travel support from Roche, Amgen and AbbVie. All other authors declare that they have no conflict of interest related to the present study.

Ethical approval This study was performed according to the ethical standards of the Ethics Committee of the Medical University of Vienna (approval no. 1166/2019) and the Helsinki Declaration of 1964 and its amendments.

Open Access This article is licensed under a Creative Commons Attribution 4.0 International License, which permits use, sharing, adaptation, distribution and reproduction in any medium or format, as long as you give appropriate credit to the original author(s) and the source, provide a link to the Creative Commons licence, and indicate if changes were made. The images or other third party material in this article are included in the article's Creative Commons licence, unless indicated otherwise in a credit line to the material. If material is not included in the article's Creative Commons licence and your intended use is not 
permitted by statutory regulation or exceeds the permitted use, you will need to obtain permission directly from the copyright holder. To view a copy of this licence, visit http://creativecommons.org/licenses/by/4.0/.

\section{References}

1. Goldberg SB, Gettinger SN, Mahajan A et al (2016) Pembrolizumab for patients with melanoma or non-small-cell lung cancer and untreated brain metastases: early analysis of a non-randomised, open-label, phase 2 trial. Lancet Oncol 17:976-983. https://doi.org/10.1016/S1470-2045(16)30053-5

2. Margolin K, Ernstoff MS, Hamid O et al (2012) Ipilimumab in patients with melanoma and brain metastases: an open-label, phase 2 trial. Lancet Oncol 13:459-465. https://doi.org/10.1016/ S1470-2045(12)70090-6

3. Long GV, Atkinson V, Lo S et al (2018) Combination nivolumab and ipilimumab or nivolumab alone in melanoma brain metastases: a multicentre randomised phase 2 study. Lancet Oncol 19:672-681. https://doi.org/10.1016/S1470-2045(18)30139-6

4. Reardon DA, Brandes AA, Omuro A et al (2020) Effect of nivolumab vs bevacizumab in patients with recurrent glioblastoma: the CheckMate 143 phase 3 randomized clinical trial. JAMA Oncol 5450:1024. https://doi.org/10.1001/jamao ncol.2020.1024

5. Bristol Myers Squibb (2019) Press release: Bristol-Myers Squibb announces phase 3 CheckMate -498 study did not meet primary endpoint of overall survival with Opdivo (nivolumab) plus radiation in patients with newly diagnosed MGMT-unmethylated glioblastoma multiforme. https://news.bms.com/press-release/corpo ratefinancial-news/bristol-myers-squibb-announces-phase-3-check mate-498-study-did. Accessed 13 Dec 2019

6. Bristol Myers Squibb (2019) Press release: Bristol-Myers Squibb provides update on phase 3 Opdivo (nivolumab) checkmate -548 trial in patients with newly diagnosed MGMT-methylated glioblastoma multiforme. https://news.bms.com/press-release/corpo ratefinancial-news/bristol-myers-squibb-provides-update-phase -3-opdivo-nivolumab-. Accessed 13 Dec 2019

7. Nduom EK, Wei J, Yaghi NK et al (2016) PD-L1 expression and prognostic impact in glioblastoma. Neuro Oncol 18:195-205. https://doi.org/10.1093/neuonc/nov172

8. Berghoff AS, Kiesel B, Widhalm G et al (2015) Programmed death ligand 1 expression and tumor-infiltrating lymphocytes in glioblastoma. Neuro Oncol 17:1064-1075. https://doi. org/10.1093/neuonc/nou307

9. Workman CJ, Rice DS, Dugger KJ et al (2002) Phenotypic analysis of the murine CD4-related glycoprotein, CD223 (LAG-3). Eur J Immunol 32:2255-2263. https://doi.org/10.1002/15214141(200208)32:8\%3c2255::AID-IMMU2255\%3e3.0.CO;2-A

10. Puhr HC, Ilhan-Mutlu A (2019) New emerging targets in cancer immunotherapy: the role of LAG3. ESMO Open 4:1-6. https:// doi.org/10.1136/esmoopen-2018-000482

11. Khair DO, Bax HJ, Mele S et al (2019) Combining immune checkpoint inhibitors: established and emerging targets and strategies to improve outcomes in melanoma. Front Immunol 10:1-20. https:// doi.org/10.3389/fimmu.2019.00453

12. Ascierto PA, Bono P, Bhatia S et al (2017) Efficacy of BMS986016, a monoclonal antibody that targets lymphocyte activation gene-3 (LAG-3), in combination with nivolumab in pts with melanoma who progressed during prior anti-PD-1/PD-L1 therapy (mel prior IO) in all-comer and biomarker-enriched popu. Ann Oncol 28:v611-v612. https://doi.org/10.1093/annonc/mdx440.011

13. Harris-Bookman S, Mathios D, Martin AM et al (2018) Expression of LAG-3 and efficacy of combination treatment with antiLAG-3 and anti-PD-1 monoclonal antibodies in glioblastoma. Int J Cancer 143:3201-3208. https://doi.org/10.1002/ijc.31661

14. Louis DN, Perry A, Reifenberger G et al (2016) The 2016 World Health Organization classification of tumors of the central nervous system: a summary. Acta Neuropathol 131:803-820. https://doi. org/10.1007/s00401-016-1545-1

15. Berghoff AS, Fuchs E, Ricken G et al (2016) Density of tumorinfiltrating lymphocytes correlates with extent of brain edema and overall survival time in patients with brain metastases. Oncoimmunology 5:e1057388. https://doi.org/10.1080/21624 02X.2015.1057388

16. Berghoff AS, Kiesel B, Widhalm G et al (2017) Correlation of immune phenotype with IDH mutation in diffuse glioma. Neuro Oncol 19:1460-1468. https://doi.org/10.1093/neuonc/nox054

17. Bender R, Lange S (2001) Adjusting for multiple testing — when and how? J Clin Epidemiol 54:343-349. https://doi.org/10.1016/ S0895-4356(00)00314-0

18. Panda A, Rosenfeld JA, Singer EA et al (2020) Genomic and immunologic correlates of LAG-3 expression in cancer. Oncoimmunology 9:1756116. https://doi.org/10.1080/21624 02X.2020.1756116

19. Matsuzaki J, Gnjatic S, Mhawech-Fauceglia P et al (2010) Tumor-infiltrating NY-ESO-1-specific CD8+ T cells are negatively regulated by LAG-3 and PD-1 in human ovarian cancer. Proc Natl Acad Sci USA 107:7875-7880. https://doi.org/10.1073/ pnas. 1003345107

20. Havel JJ, Chowell D, Chan TA (2019) The evolving landscape of biomarkers for checkpoint inhibitor immunotherapy. Nat Rev Cancer 19:133-150. https://doi.org/10.1038/s41568-019-0116-x

21. Lim M, Ye X, Piotrowski AF et al (2020) Updated safety phase I trial of anti-LAG-3 alone and in combination with anti-PD-1 in patients with recurrent GBM. J Clin Oncol 38:2512. https://doi. org/10.1200/JCO.2020.38.15_suppl.2512

22. Fröhlich A, Sirokay J, Fietz S et al (2020) Molecular, clinicopathological, and immune correlates of LAG3 promoter DNA methylation in melanoma. EBioMedicine 59:1-15. https://doi. org/10.1016/j.ebiom.2020.102962

Publisher's Note Springer Nature remains neutral with regard to jurisdictional claims in published maps and institutional affiliations. 